\title{
Circulating Immune Biomarkers in Peripheral Blood Correlate With Clinical Outcomes in Advanced Breast Cancer
}

\section{Natalia Palazón-Carrión}

Virgen Macarena University Hospital

Carlos Jiménez-Cortegana

Virgen Macarena University Hospital

M. Luisa Sánchez-León

Virgen Macarena University Hospital

\section{Fernando Henao-Carrasco}

Virgen Macarena University Hospital

Esteban Nogales-Fernández

Virgen Macarena University Hospital

\section{Massimo Chiesa}

Spanish Breast Cancer Group

\section{Rosalía Caballero}

Spanish Breast Cancer Group

\section{Federico Rojo}

IIS-Fundacion Jimenez Diaz-CIBERONC

María-Adoración Nieto-García

University of Seville

Víctor Sánchez-Margalet

Virgen Macarena University Hospital

Luis de la Cruz-Merino ( $\square$ Idelacruzmerino@gmail.com )

Virgen Macarena University Hospital

\section{Research Article}

Keywords: MDSCs, regulatory T cells, OX40, peripheral blood, advanced breast cancer

Posted Date: February 23rd, 2021

DOI: https://doi.org/10.21203/rs.3.rs-228062/v1 
License: (c) (i) This work is licensed under a Creative Commons Attribution 4.0 International License. Read Full License

Version of Record: A version of this preprint was published at Scientific Reports on July 13th, 2021. See the published version at https://doi.org/10.1038/s41598-021-93838-w. 

ADVANCED BREAST CANCER.

\author{
AUTHORS: Natalia Palazón-Carrión ${ }^{1}$, Carlos Jiménez-Cortegana르, M. Luisa Sánchez-León ${ }^{1}$, Fernando Henao- \\ Carrasco $^{1}$, Esteban Nogales-Fernández ${ }^{1}$, Massimo Chiesa ${ }^{3}$, Rosalía Caballero ${ }^{3}$, Federico Rojo ${ }^{4}$, María-Adoración \\ Nieto-García ${ }^{5}$, Víctor Sánchez-Margalet ${ }^{2 *}$, Luis de la Cruz-Merino ${ }^{1,3,6^{*}}$.
}

*CORRESPONDING AUTHOR: Luis de la Cruz-Merino (ORCID iD https://orcid.org/0000-0002-5333-0535)

On behalf of the Spanish Breast Cancer Group (GEICAM) and the Spanish Group for Immunobiotherapy of Cancer (GÉTICA)

${ }^{1}$ Clinical Oncology Department. Virgen Macarena University Hospital, Seville, Spain

${ }^{2}$ Department of Medical Biochemistry and Molecular Biology, and Immunology, School of Medicine. Virgen Macarena University Hospital, University of Seville, Spain

${ }^{3}$ GEICAM (Spanish Breast Cancer Research Group), Madrid, Spain

${ }^{4}$ Pathology Department. IIS-Fundacion Jimenez Diaz-CIBERONC, Madrid, Spain Funding: PT17/0015/0006, PI1800382

${ }^{5}$ Department of Preventive Medicine and Public Health. Faculty of Medicine. University of Seville

${ }^{6}$ Faculty of Medicine. University of Seville

\title{
ACKNOWLEDGMENTS
}

We thank the patients and their families for their commitment; the women from the healthy cohort for their voluntariness; the nursery team from the Research Unit of the UHVM Clinical Oncology Department for collecting the peripheral blood samples; the patients associations "Ana María Carmona", "Todo Suma", "Verenia Santa Agueda", "AMAMA", and "Sandra Ibarra Foundation" for their collaboration; the entire Clinical Oncology Department and the Day Care Unit of the Virgen Macarena University Hospital.

\section{Declarations}

Funding

Research was partially supported by a grant from the Andalusian Public Foundation Progress and Health (PI0502-2014 FPS-2014).

Conflicts of interest/Competing interests

Authors declare no potential conflicts of interest with respect to this study

Ethics approval

Protocol was approved by the VMUH's institutional review board (ref. LCM-INM-2015-01/Law 14/2007, of July 3) according to the ethical principles included in Declaration of Helsinki 1964 (2013 update)

Consent to participate

All the patients signed written informed consent to participate in this study.

Consent for publication

All the authors gave their consent to submit this version of the manuscript

Availability of data and material

Data and materials are accessible under formal request

Code availability (software application or custom code)

Not applicable

Authors' contributions

All the authors participated in the following tasks of the work:

1. Substantial contributions to conception and design, acquisition of data, and/or analysis and interpretation of results.

2. Substantial scientific and intellectual contributions to the drafting or rewriting of the initial and/or revised manuscript.

3. Approval of the final accepted version of the manuscript. 


\section{ABSTRACT}

Identification of the different elements intervening at the immunoedition process, in each body compartment, seems key to explain clinical evolution in several tumor types. In this study, a set of immune biomarkers (myeloid derived suppressor cells, regulatory T cells, and OX40+ and PD-1+ T lymphocytes counts) in peripheral blood of patients diagnosed with advanced breast cancer, were analyzed prior to and along the implementation of first line antineoplastic therapy. Subsequently, a formal comparison between groups with clinical benefit versus progression of disease and with a healthy women cohort was executed.

Results reflected that patients basally showed higher levels of myeloid derived suppressor cells $(35.43, I R=180.73$ vs $17.53, I R=16.96$ cells $/ \mu l ; p=0.001)$ and regulatory T cells $(32.05, I R=29.84$ vs $22.61, I R=13.57$ cells $/ \mu l ; p=0.001)$ in comparison with healthy women. Furthermore, after therapy, an increase in the number of activated $T$ lymphocytes (expressing OX40), and a decrease of immune inhibitory cells (MDSCs, and Tregs), and the number of inhibited (or exhausted) T lymphocytes (expressing PD-1), could be ascertained in patients with clinical benefit $(p \leq 0.001)$. The opposite trend was observed in the case of disease progression. These findings suggest that some critical immune elements can be easily detected and measured in peripheral blood, which open a new opportunity for translational research, as they seem to be clearly correlated with clinical evolution, at least in $A B C$.

Key words: MDSCs, regulatory T cells, OX40, peripheral blood, advanced breast cancer.

\section{INTRODUCTION}

Breast cancer $(\mathrm{BC})$ is the leading cause of cancer in women with more than 2 million of new cases worldwide every year. At present, although it is considered that $80-85 \%$ of the $\mathrm{BC}$ patients can be cured, a sizeable proportion recurs in an advanced stage and eventually die as a consequence of the disease[1].

Immunoedition phenomenon has been postulated as a crucial event in BC initiation and progression, and this condition can be detected and revealed through a wide set of assays, both in the tumor and peripheral blood[2]. Immunoediting hypothesis recognizes the crucial role of the different elements of tumor microenvironment (TME), with respect to development and progression of tumors, including breast cancer[3,4]. Consequently, the balance between immune inhibitory and stimulatory signals is determinant to explain the final effect, anergy or activation of the immune system against cancer cells. Density and function of some cell populations seem extraordinary relevant in this context and, interestingly, they can be detected and measured not just in TME but even in peripheral blood, what renders many advantages over tissue biopsies. At this point, myeloid derived suppressor cells (MDSCs) and different lymphocyte subpopulations, such as regulatory T cells (Tregs) [5]' activated CD4+ and CD8+ T lymphocytes (expressing OX-40) or inhibited T lymphocytes (expressing PD-1) [6,7], and other subset of cells can be measured in peripheral blood of patients with advanced breast cancer (ABC) $[3,4,8-10]$.

MDSCs constitute a population of immature myeloid cells with an extensive variety of immunosuppressive properties[11]. Their role in cancer has been recently elucidated, demonstrating in different studies that higher levels of MDSCs represent an adverse prognostic factor[12-14]. The main mechanisms for immunosuppression that MDSCs set up are the following: alteration of L-arginine metabolism through Arginase-1 (Arg-1) production and via iNOS allowing NO generation, promotion of cysteine uptake, stimulation of Tregs and tumor-associated macrophages type-2 (TAM-2) production, and inhibition of CD8+ T lymphocytes and natural killer T (NKT) function[15].

MDSCs can be detected in peripheral blood in cancer patients, especially in advanced stages. However, due to the intrinsic heterogeneity of these cells, analytical characterization has been challenging. At present, MDSCs are generally defined by CD45+CD3-CD19-CD20-CD56-CD16-HLADR-CD33+CD11b+ expression through flow cytometry, being CD14+ and CD15+ subsets assigned as monocytic and granulocytic MDSCs, respectively[16].

In the present work we aimed to analyze circulating MDSC levels from ABC patients in a basal state immediately after diagnosis of metastatic disease and along treatment implementation. Subsequently, a formal comparison between groups with clinical benefit (CB) versus progression of disease (PD) was executed. Other lymphocyte subpopulations were analyzed, including immunosuppressive Tregs, activated (OX-40+) and inhibited (PD-1+) CD4+ and CD8+ T lymphocytes. In addition, a healthy women (HW) cohort was included as control group. 


\section{MATERIALS AND METHODS}

Patients consecutively diagnosed of $A B C$ and $\mathrm{HW}$ were invited to participate in the study at Virgen Macarena University Hospital (VMUH) in Seville (Spain). Protocol was approved by the VMUH's institutional review board (ref. LCM-INM-2015-01/Law 14/2007, of July 3) according to the ethical principles included in Declaration of Helsinki 1964 (2013 update) [17]. Research was partially supported by a grant from the Andalusian Public Foundation Progress and Health (PI-0502-2014 FPS-2014). All the patients gave written informed consent to participate in this study.

Patients received systemic therapies following local protocols based on International Guidelines (NCCN[18] and $A B C$ Guidelines[19]). Clinical response was assigned following Response Evaluation Criteria in Solid Tumors version 1.1 (RECIST v 1.1.) [20] considering Clinical Benefit (CB) the sum of Complete Responses (CR), Partial Responses (PR) and Stabilization of Disease (SD). ABC patients were classified in luminal A/B, triple negative and HER2+ subtypes, by immunohistochemistry criteria according to the established St Gallen international guidelines[21].

\section{Flow cytometric analyses in whole blood samples.}

Peripheral blood samples were collected from subjects in EDTA-K3 tubes before treatment onset and pre-dose to cycle 3 and cycle 6, to determine MDSCs, Tregs, and OX40+ and PD-1+ T lymphocytes (TL) counts. Cell populations were determined by flow cytometry of whole blood using the BD FACSCanto ${ }^{\text {TM }}$ flow cytometry system. M-MDSCs were determined as CD45+CD11b+CD33+HLA-DR-CD14+CD15-, G-MDSC as CD45+CD11b+CD33+HLA-DR-CD14-CD15+, Tregs as CD3+CD4+CD25+CD127-, activated TL were determined as CD3+CD4+OX40+ TL and CD3+CD8+OX-40+ TL, inhibited TL as CD3+CD4+OX-40-PD-1+ and CD3+CD8+OX-40-PD1+. Leukocyte and lymphocyte counts were obtained from hemotologic count (Sysmex CS-1000).

\section{Monoclonal antibodies.}

Antibodies were obtained from Becton Dickinson Immunocytometry Systems (BDIS, San Jose, CA, USA) and were used at the manufacturer's recommended concentrations.

MDSCs: PerCP-Cy5.5 Mouse Anti-Human CD 45 (ref no. 564105), APC-Cy7 Rat Anti-CD11b (ref no. 557657), PE Mouse Anti-Human CD 33 (ref no. 555450), PE-Cy7 Mouse Anti-Human HLA-DR (ref no. 560651), FITC Mouse Anti-Human CD 14 (ref no. 555397) and APC Mouse Anti-Human CD 15 (ref no. 551376).

Treg kit (ref no. 560249), including FITC anti-Human CD4, PE-Cy7 anti-Human CD25, and Alexa-Fluor 647 antiHuman CD127.

Activated and inhibited T Lymphocytes: FITC Mouse Anti-Human OX-40 (CD134) ref no. 555837, PE Mouse AntiHuman CTLA-4 (CD152) (ref no.555853), PerCP-Cy5.5 Mouse Anti-Human CD8 (ref no. 565310), PE-Cy7 Mouse Anti-Human CD4 (ref no. 557852), APC Mouse Anti-Human PD-1 (CD279) (ref no. 558694), APC-H7 Mouse AntiHuman CD3 ref no. 560176).

\section{Data analysis.}

Statistical analysis was performed by SPSS 26.0 software package (SPSS Inc., Chicago, IL, USA). Normal distribution of analysed variables was checked by watching histogram, box plot, Q-Q plot- and the outcomes of normality tests of Kolmogorov-Smirnov (samples $\geq 50$ ) and Shapiro-Wilk (samples<50). Due to absence of normality, the lymphocytes distributions before and after systemic therapies were compared using nonparametric Wilcoxon test for related pairs. Lymphocytes distributions from CB vs PD were compared using nonparametric U-Mann-Whitney test for independent samples. Basal lymphocytes distributions from $A B C$ patients versus $\mathrm{HW}$ were also compared using non-parametric U-Mann-Whitney test. Statistically significant differences were considered at the $95 \%$ level of confidence $(p \leq 0.05)$.

\section{RESULTS}

From 15 January 2016 to 13 February 2020 a total of $20 \mathrm{HW}$ and 51 ABC patients signed informed consent and were consecutively recruited to participate in the present study. Main clinico-pathological characteristics can be summarized as follows: most of the patients (82\%) were included in the luminal A + Luminal B (her2-ve) BC entities. With respect to $1^{\text {st }}$ line treatment, $61 \%$ were treated with cyclin-dependent kinase $4 / 6$ inhibitors plus hormonotherapy, $18 \%$ with hormonotherapy, $16 \%$ received chemotherapy, $6 \%$ were treated with anti-her2 monoclonal antibodies and 2\% with chemoimmunotherapy. By 13 February 2020, the following basal measurements from peripheral blood were taken: CD8+ T and CD4+ T lymphocytes with expression of OX-40, 
CD8+ $T$ and CD4+ T lymphocytes with expression of PD-1, Tregs and MDSC levels. Furthermore, in ABC patients these levels were determined in cycle 3 (C3) and cycle 6 (C6) to monitor their evolution. In C3, measurements were undertaken in 45 patients since three of them died due to PD; in two patients, response status could not be established by RECIST 1.1 criteria, and finally in three women C3 extraction had not yet be done due to SARSCoV-2 (COVID-19) pandemic. C6 analysis could be performed in 40 patients since one of them passed away due to clinical PD and in another four patients last extraction had not yet be done either for the same reason as in C3. Objective response by RECIST 1.1 criteria could be established in 48 patients after using computed tomography (CT) in C3 (+/- 30 days) Figure 1.

\section{Clinical significance in $A B C$ of circulating MDSC}

\subsection{MDSC levels are increased in $A B C$ patients}

Baseline MDSC levels before starting treatment in $A B C$ patients (median cells/ $\mu$ l, 35.43; interquartile range $(I R)=180.73)$ were higher than in HW (median, 17.53; IR=16.96) $(p=0.001)$.

In addition, basal levels of granulocytic and monocytic MDSCs were also significantly higher in ABC patients compared to HW ( $\mathrm{p} \leq 0.035)$. Medians were 18.46 (IR=30.67) cells $/ \mu \mathrm{l}$ in $\mathrm{ABC}$ vs $10.28(\mathrm{IR}=17.48)$ cells/ $\mu \mathrm{l}$ in $\mathrm{HW}$ for M-MDSC and $11.38(I R=57.60)$ cells/ $\mu \mathrm{l}$ in ABC vs $4.99(I R=16.96)$ cells/ $\mu \mathrm{l}$ in HW for G-MDSC. Table 1; Figure 2.

\subsection{Low circulating MDSC levels are associated with clinical benefit}

MDSC, M-MDSC and G-MDSC levels decreased along treatment in $A B C$ patients with $C B$ whilst they increased in those with $P D$, being the difference statistically significant $(p \leq 0.004)$ in $A B C$ patients with $C B$ vs PD at C6.

Medians of MDSC were $33.63(I R=39.34)$ at baseline vs $10.36(I R=15.84)$ cells $/ \mu l$ at $C 6$ in $A B C$ patients with $C B$ while they were $37.48(I R=20.63)$ at baseline vs $78.54(I R=128.63)$ cells/ $\mu$ lat $C 6$ in $A B C$ patients with PD. Medians of M-MDSC were $18.46(I R=33.49)$ at baseline vs $4.15(I R=6.48)$ cells/ $\mu$ lat $C 6$ in $A B C$ patients with $C B$ while were $16.20(I R=22.97)$ at baseline vs $42.04(I R=86.99)$ cells/ $\mu$ la $C 6$ in $A B C$ patients with PD. Medians of $G-M D S C$ were $12.74(\mathrm{IR}=18.14)$ at baseline vs $3.36(\mathrm{IR}=7.03)$ cells/ $\mu \mathrm{l}$ at $\mathrm{C} 6$ in $\mathrm{ABC}$ patients with $\mathrm{CB}$ while they were 14.20 $(I R=28.56)$ at baseline vs $10.00(I R=24.23)$ cells/ $\mu$ lat $C 6$ in $A B C$ patients with PD. Tables 2-3; Figure 3.

\section{OX-40 expression from CD4+ T and CD8+ T lymphocytes in ABC}

\subsection{Increased $O X-40+T$ lymphocyte levels are detected in $A B C$ patients}

Basal total OX-40+, CD4+ T and CD8+ T lymphocyte levels before starting treatment in $A B C$ patients were higher than in HW. Medians of total OX-40+ T lymphocyte were $9.89(\mathrm{IR}=28.71)$ cells/ $\mu \mathrm{l}$ in ABC patients vs 3.01 (IR=3.01) cells $/ \mu$ in $\mathrm{HW}$, being the difference statistically significant $(p=0.001)$. Table 1; Figure 2.

\subsection{Increased $O X-40+T$ lymphocyte levels in $A B C$ patients are correlated with clinical benefit.}

$O X-40+, C D 4+O X-40+$ and $C D 8+O X-40+T$ levels increase along treatment in $A B C$ patients with $C B$ while decrease in those with PD. Values at baseline vs $C 6$ showed statistically significant differences $(p \leq 0.009)$ in $A B C$ patients with $\mathrm{CB}$ but not in PD.

Medians of OX-40+ T lymphocyte levels in ABC patients with $C B$ were $9.89(I R=28.64)$ at baseline vs 37.28 $(\mathrm{IR}=43.54)$ cells/ $\mu \mathrm{l}$ at $\mathrm{C6}$; $C D 4+O X-40+T$ were $8.32(\mathrm{IR}=25.46)$ at baseline vs $33.22(\mathrm{IR}=40.49)$ cells $/ \mu \mathrm{l}$ at $\mathrm{C} 6$, and CD8+OX-40+ T were $1.85(\mathrm{IR}=2.37)$ at baseline vs $2.63(\mathrm{IR}=5.72)$ cells/ $\mu \mathrm{l}$ at $\mathrm{C6}$. Tables 2-3; Figure 3.

\section{PD-1 expression from CD4+ $T$ and CD8+ T lymphocytes in $A B C$}

\subsection{PD-1+ T lymphocyte levels decrease in ABC patients}

Basal total PD-1+ T and CD4+PD-1+ T lymphocyte levels before starting treatment in ABC patients were slightly lower than in HW, but differences did not reach statistical significance. Medians of total PD-1+ T lymphocytes were 10.77 (IR=8.55) cells/ $\mu \mathrm{l}$ in $A B C$ patients vs 13.00 (IR=6.19) cells/ $\mu \mathrm{l}$ in HW, ( $p=0.245)$. Table 1; Figure 2.

\subsection{Decreased PD-1+ T lymphocyte levels in $A B C$ patients predict clinical benefit.}

PD-1+, CD4+PD-1+ and CD8+PD-1+ T levels diminished along treatment in ABC patients regardless of response, however values at baseline vs $C 6$ showed statistically significant differences $(p \leq 0.001)$ only in $A B C$ patients with $\mathrm{CB}$ but not in PD. Medians of PD-1+ T lymphocyte levels in $\mathrm{ABC}$ patients with $\mathrm{CB}$ were $11.16(\mathrm{IR}=8.99)$ at baseline vs $4.82(\mathrm{IR}=3.74)$ cells/ $\mu \mathrm{l}$ at $\mathrm{C} 6$; $C D 4+P D-1+\mathrm{T}$ were $6.95(\mathrm{IR}=5.08)$ at baseline vs $2.93(\mathrm{IR}=3.12)$ cells/ $\mu \mathrm{l}$ at $\mathrm{C} 6$, and CD8+OX-40+ T were $3.62(\mathrm{IR}=2.45)$ at baseline vs $1.51(\mathrm{IR}=1.05)$ cells/ $\mu \mathrm{l}$ at $\mathrm{C6}$. Tables 2-3; Figure 3.

\section{Levels of regulatory $T$ cells (Tregs) in ABC}




\subsection{Tregs levels are increased in $A B C$ patients}

Basal Tregs levels before starting treatment in $A B C$ patients were higher than in $\mathrm{HW}$ with a median of 32.05 $(I R=29.84)$ vs $22.61(I R=13.57)$ cells $/ \mu l$, being the difference statistically significant $(p=0.001)$. Table 1; Figure 2 .

\subsection{Decreased Tregs levels are observed in $A B C$ patients with clinical benefit}

Tregs levels decreased along treatment in $A B C$ patients, being the difference statistically significant $(p \leq 0.001)$ in $\mathrm{ABC}$ patients with $\mathrm{CB}$.

Medians of Tregs were 31.87 (IR=27.87) at baseline vs $19.01(\mathrm{IR}=16.64)$ cells $/ \mu \mathrm{I}$ at $C 6$ in $A B C$ patients with $C B$ while they were $39.30(I R=52.24)$ at baseline vs $28.36(I R=32.03)$ cells/ $\mu$ lat $C 6$ in $A B C$ patients with PD. Tables 23; Figure 3.

\section{DISCUSSION}

In the last few years, growing translational evidence has definitively established immunoediting as a prominent hallmark in BC that should not be overlooked[22-27]. Relationship between the presence of TILs and MDSCs in the tumoral microenvironment (TME) and the response to treatment and survival of patients has been described. Thus, in certain subtypes of breast cancer, i.e. triple-negative and HER2 breast subtypes, a higher presence of TILs in TME implies improved results in pathological complete responses in the neoadjuvant setting and an impact in survival is suggested, although clinical evidence at this point is more debatable[23,28]. A relation between the presence of elevated circulating MDSCs and prognosis have been clearly established, being associated with advanced cancer stage, higher tumor burden, lower progression-free survival (PFS) and overall survival (OS) rates[14, 29-32] as well as with the response to cancer treatment by decreasing the efficacy of chemotherapy, radiotherapy, immunotherapy or other targeted therapies[33-35].

MDSCs represent a heterogeneous population of immature myeloid cells among which we can distinguish two main types based on their phenotype and morphology: the granulocytic subtype (G-MDSC) and the monocytic subtype (M-MDSC). In our research, we have used the following surface markers to identify them: CD45+CD11b+CD33+ HLA-DRlow/- in addition to CD14-CD15+ for G-MDSCs and CD14+CD15- for M-MDSC[16]. Slightly higher levels of M-MDSC over G-MDSCs have been observed in our patients. M-MDSC is a subtype that seems to be related with a greater immunosuppressive role, through NO generation, in comparison with GMDSC[36]

In physiological conditions, the granulocyte-macrophage colony-stimulating factor (GM-CSF) induces myelopoiesis while the granulocyte colony-stimulating factor (G-CSF) and the macrophage colony-stimulating factor (M-CSF) stimulate the differentiation of granulocytes and macrophages in response to pathogen-derived signals like Toll-like receptors (TLR) ligands, damage-associated molecular patterns (DAMPs) and pathogenassociated molecular pattern (PAMPs) molecules. These events trigger the mobilization of neutrophils and monocytes with the production of inflammatory cytokines and costimulatory molecules. In pathological conditions of chronic inflammation or cancer, the myelopoiesis continues and is characterized, among other events, by the overproduction of MDSCs[37]. Firstly, the expansion phase of immature myeloid cells takes place through the influence of factors produced by the tumor stroma or the bone marrow like GM-CSF, G-CSF, M-CSF, stem cell factor (S-SCF) or the vascular endothelial growth factor (VEGF). Secondly, the suppressing action of MDSC is executed by the production of reactive oxygen species (ROS), cytokines (TGF- $\beta$, IL-10, IL-6), the expression of enzymes such as indoleamine 2,3-dioxygenase (IDO) or arginase-1 (ARG1) and the production of prostaglandin E2 (PGE2) [38,39].

At the TME level, tumors that induce expansion of MDSCs in their surrounding stroma contribute to the tumorinduced immune suppression mechanisms by inhibiting the effector function of $\mathrm{T}$ cells and natural killer cells through several cellular exhaustion and inactivation mechanisms[40], which are enhanced by the increased production of Tregs. The generation of ROS impair recognition through the class II major histocompatibility complex (MHC II) while nitric oxide (NO) inhibits the IL-2 receptor (IL-2R) that, in turn, affects negatively signaling via T cell receptor (TCR). Furthermore, there is a depletion of $L$-arginine due to the expression of high levels of ARG1 which leads to a decrease of the average life of mRNA in the CD3 $\zeta$ chain of T cells. Aforementioned events, added to decreased secretion of IFN- $\gamma$ and granzyme B, damage the activity of effector T lymphocytes, and may even lead to their apoptosis[41].

In our research, $A B C$ patients showed increased levels of MDSCs and Tregs in peripheral blood in comparison with healthy women. Therefore, it is plausible to consider that immune profile of the patients is impaired, 
facilitating an immune microenvironment that favors the tumor development and progression, as the escape phase of the immunoedition hypothesis dictates. Even more interesting, patients that received cancer treatment and experienced clinical benefit, whether this was a partial or complete response or stabilization of the disease, experienced a drastically drop in MDSCs and Tregs levels, whilst those patients in which disease progressed showed a dramatically increase in MDSCs levels. These findings strengthen the immunoedition postulates in BC, and suggest that this could be a reversible process, as immune profiling of the patients improve when disease back to be under control.

The immunosuppressive role of MDSCs and Tregs and their influence in the anergy of T cells is remarkable in the tumoral microenvironment[42]. The greater expression of immune checkpoint molecules like PD-1/PDL-1 to damage or delay the immune response by inhibiting $T$ cells or the expression of immune system co-stimulators such as $0 X-40$ is remarkable too. These biomarkers seem potential promissory targets for an outstanding performance of immunotherapy and personalized therapy[24]. Furthermore, some new emerging data suggest that the presence of IDO and ARG-1 by MDSCs upregulates the expression of PD-1 over MDSCs, that is, by blocking PDL-1 we would manage to mitigate the activity of MDSCs[43].

In our work, we could demonstrate enhanced levels of CD4+ and CD8+ T lymphocytes with expression of OX-40 in $A B C$ patients compared to healthy women. However, it was in the healthy cohort where we observed a higher percentage of $\mathrm{T}$ lymphocytes with expression of the co-repressive molecule PD-1, which reveal the great complexity of the mechanisms of interrelation among the immune system elements beyond those described here: the release of inhibitory cytokines, the expansion of T regulators and tumor-associated macrophages (TAM), the expression of immune checkpoint and the presence of MDSCs working all together to create this immunosuppressive microenvironment $[3,15,34-36]$. Nonetheless, with respect to CD4+ T and CD8+ T expressing OX-40, we could observe an exceptional rise in these cells in the ABC cohort that reached CB. On the contrary, patients that progressed showed declining and lower levels of OX40 T cells. Furthermore, a significant decrease in $C D 4+T$ and $C D 8+T$ with expression of PD-1 along treatment in $A B C$ patients with $C B$ could be ascertained.

These findings seem quite promising since they highlight the undeniable immunosuppressive role of MDSCs and the co-repressive/co-stimulatory activity of PD-1/OX-40 molecules, respectively. The depletion in the levels of MDSC, Tregs and the increase of OX-40 along antineoplastic treatment, whether this is hormonotherapy alone, chemotherapy, hormonotherapy plus cyclin-dependent kinase inhibitors 4/6, or other combined strategies, could improve immune profile of the patients reverting their immunosuppressive status with respect to cancer cells. These determinations could also become potential biomarkers to monitor in a simple way by means of a simple peripheral blood extraction the response to treatment.

Several combinations of treatments are being carried out in preclinical studies that target MDSC and immune checkpoint, boosting the immune effector activity in cancer. Recently, it has been revealed that the inhibition of $\mathrm{PI3K}$, JAK/STAT and CDK4/6 pathways improve the infiltration of cytotoxic CD8+ T type TILs in mouse models with triple-negative breast cancer. This effect seems tightly related to the decrease of the MDSCs levels, what ultimately facilitate the action of effector T cells. In top of that, this effect may be boosted by blocking some specific immune checkpoint inhibitors[44]. Consequently, targeting MDSCs aimed to deplete them can potentially increase the efficacy of the modern immune checkpoint inhibitors currently in use for several oncological diseases[45].

Results of this study put the focus on potential biomarkers easily detectable in peripheral blood to monitor the response of antineoplastic treatments in $\mathrm{ABC}$. Likewise, it permits foresee new targets for immunomodulatory strategies in breast cancer. There are uncountable therapeutic possibilities at this point, since there are different options to boost the immune response of the host whether employing classic agents (doxorubicin, cyclophosphamide, gemcitabine, and so on) or newer agents such as anti-PD-1 (antibodies that block the inhibiting and proapoptotic effect of their signals in T cells), anti-OX-40 or anti-CD40 (antibodies that stimulate the activating signaling of T lymphocytes) and/or within the context of combinations.

Although data from this work seem clear, additional confirmatory studies with higher sample size and/or with other tumor types are obviously needed. Nonetheless, new avenues of research can be suggested from these findings since a sort of immune liquid biopsy might proportionate extraordinarily valuable and complementary information in BC, not only in the advanced setting but even also during follow-up of BC patients previously treated with curative purposes. 


\section{REFERENCES}

1. Siegel RL, Miller KD, Jemal A (2019) Cancer statistics. CA Cancer J Clin 2019; 69:7.

2. Remon J, García-Campelo R, de Álava E, et al (2019) Liquid biopsy in oncology: a consensus statement of the Spanish Society of Pathology and the Spanish Society of Medical Oncology. Clin Transl Oncol . https://doi.org/10.1007/s12094-019-02211-x.

3. De la Cruz-Merino L, Barco-Sánchez A, Henao Carrasco F, et al (2013) New insights into the role of the immune microenvironment in breast carcinoma. Clin Dev Immunol. 2013:785317.

4. de la Cruz-Merino L, Grande-Pulido E, Albero-Tamarit A, et al (2008). Cancer and immune response: old and new evidence for future challenges. Oncologist (12):1246-54.

5. Fangxuan Li, Yang Zhao, Lijuan Wei et al (2018). Tumor-infiltrating Treg, MDSC, and IDO expression associated with outcomes of neoadjuvant chemotherapy of breast cancer. Cancer Biol Ther. 19(8): 695-705.

6. Deng JD, Zhao SD, Zhang XD, et al (2019) OX40 (CD134) and OX40 ligand important immune checkpoints in cancer. Oncotargets and Therapy. 12:7347-7353.

7. Schütz FD, Stefanovic SD, Mayer L, et al (2017). PD-1/PD-L1 pathway in breast cancer. Oncol Res Treat. 40(5):294-297.

8. de la Cruz-Merino L, Chiesa M, Caballero R, et al (2017). Breast Cancer Immunology and Immunotherapy: Current Status and Future Perspectives. Int Rev Cell Mol Biol. 331:1-53.

9. de la Cruz-Merino L, Palazón-Carrión N, Henao-Carrasco F, et al (2019). New Horizons in Breast Cancer: The Promise of Immunotherapy. Clin Transl Oncol.21(2):117-125.

10. Ali HR, Provenzano E, Dawson SJ, et al (2014). Association between CD8+ T-cell infiltration and breast cancer survival in 12439 patients. Ann Oncol. 25(8):1536-43.

11. Tesi RJ (2019). Trends Pharmacol Sci. 40(1):4-7.

12. Markowitz J, Wesolowski R, Papenfuss T, et al (2013). Myeloid-derived suppressor cells in breast cancer. Breast Cancer Res Treat. 140(1):13-21.

13. Cole S, Montero A, Garret-Mayer E, et al (2009). Elevated circulating myeloid derived suppressor cells (MDSC) are associated with inferior overall survival and correlate with circulating tumor cells (CTC) in patients with metastatic breast cancer. Cancer Research. 69(24 Supplement).

14. Diaz-Montero CM, Salem ML, Nishimura MI, et al (2009). Increased circulating myeloid-derived suppressor cells correlate with clinical cancer stage, metastatic tumor burden, and doxorubicin-cyclophosphamide chemotherapy. Cancer Immunol Immunother. 58: 49-59.

15. Gabrilovich DI, Nagaraj S (2009) Myeloid-derived-suppressor cells as regulators of the immune system. Nat Rev Immunol. 9(3): 162-174.

16. Ma P, Beatty PL, McKolanis J, et al (2019). Circulating Myeloid Derived Suppressor Cells (MDSC) That Accumulate in Premalignancy Share Phenotypic and Functional Characteristics with MDSC in Cancer. Front. Immunol. 10:1401.

17. World medical association, World medical association declaration of Helsinki ethical principles for medical research involving human subjects. Adopted by 64th WMA general Assembly, Fortaleza, Brazil, October 2013. JAMA 2013: 310(20): 2191-4.

18. Gradishar WJ, Anderson BO, Abraham J, et al (2020). Breast Cancer, Version 3.2020, NCCN Clinical Practice Guidelines in Oncology. J Natl Compr Canc Netw. Apr;18(4):452-478.

19. Cardoso F, Senkus E, Costa A, et al (2018). 4th ESO-ESMO International Consensus Guidelines for Advanced Breast Cancer (ABC 4). Ann Oncol. 29: 1634-165.

20. Eisenhauer EA, Therasse $P$, Bogaerts J, et al (2009). New response evaluation criteria in solid tumours: revised RECIST guideline (version 1.1) Eur J Cancer. 45:228-47.

21. Goldhirsch A, Winer EP, Coates AS, et al (2013). Personalizing the treatment of women with early breast cancer: highlights of the St Gallen International Expert Consensus on the Primary Therapy of Early Breast Cancer 2013. Ann Oncol. 24(9):2206-23.

22. Denkert C, Loibl S, Noske A, et al (2010). Tumor-associated lymphocytes as an independent predictor of response to neoadjuvant chemotherapy in breast cancer. Journal of Clinical Oncology. 28:105-113.

23. Issa-Nummer Y, Darb-Esfahani S, Loibl S, et al (2013). Prospective Validation of Immunological Infiltrate for Prediction of Response to Neoadjuvant Chemotherapy in HER2-Negative Breast Cancer - A Substudy of the Neoadjuvant GeparQuinto Trial. PLoS ONE 8(12): e79775.

24. Schmid P, Adams S, Rugo HS, et al (2018). Atezolizumab and Nab-Paclitaxel in Advanced Triple-Negative Breast Cancer. N Engl J Med. 379(22):2108-2121.

25. Alvarez R, Oliver L, Valdes A, et al (2018). Cancer-induced systemic myeloid dysfunction: Implications for treatment and a novel nanoparticle approach for its correction. Seminars in Oncology. 45:84-94. 
26. Fernandez A, Oliver L, Alvarez R, et al (2014). Adjuvants and myeloid-derived suppressor cells: Enemies or allies in therapeutic cancer vaccination. Human Vaccines \& Immunotherapeutics 10:11, 3251-3260.

27. Sánchez-Margalet V, Barco-Sánchez A, Vilariño-García T, et al (2019). Circulating regulatory T cells from breast cancer patients in response to neoadjuvant chemotherapy. Transl Cancer Res. 8 - 1, pp. 59 - 65. $02 / 2019$. ISSN 2218-676X

28. Cimino-Mathews A, Thompson E, Taube JM, et al (2016) PD-L1 (B7-H1) expression and the immune tumor microenvironment in primary and metastatic breast carcinomas. Human Pathol. 47:52-63.

29. Yu J, Du W, Yan F, et al (2013). Myeloid-Derived Suppressor Cells Suppress Antitumor Immune Responses through IDO Expression and Correlate with Lymph Node Metastasis in Patients with Breast Cancer. J Immunol 190 (7) 3783-3797.

30. Wu WC, Sun HW, Chen HT, et al (2014). Circulating hematopoietic stem and progenitor cells are myeloidbiased in cancer patients. Proc Natl Acad Sci U S A. 111(11):4221-6.

31. Yang G, Shen W, Zhang Y, et al (2017). Accumulation of myeloid-derived suppressor cells (MDSC) induced by low levels of IL-6 correlates with poor prognosis in bladder cancer. Oncotarget. 8(24):38378-38388.

32. Jordan KR, Amaria RN, Ramirez O, et al (2013). Myeloid-derived suppressor cells are associated with disease progression and decreased overall survival in advanced-stage melanoma patients. Cancer Immunol Immunother. 62(11):1711-1722.

33. Kawano M, Mabuchi S, Matsumoto Y, et al (2015). The significance of G-CSF expression and myeloid-derived suppressor cells in the chemoresistance of uterine cervical cancer. Scientific reports. 5:18217.

34. Wang D, An G, Xie S, Yao Y, Feng G (2016). The clinical and prognostic significance of CD14(+) HLA-DR(-/low) myeloid-derived suppressor cells in hepatocellular carcinoma patients receiving radiotherapy. Tumour Biol. 37(8):10427-10433.

35. Weber J, Gibney G, Kudchadkar R, et al (2016). Phase I/II Study of Metastatic Melanoma Patients Treated with Nivolumab Who Had Progressed after Ipilimumab. Cancer Immunol Res. 4(4):345-353.

36. Veglia F, Perego M, Gabrilovich D (2018). Myeloid-derived suppressor cells coming of age. Nat Immunol. 19:108-19.

37. Gabrilovich DI, Ostrand-Rosenberg S, Bronte V (2013). Coordinated regulation of myeloid cells by tumours. Nat Rev Immunol. 12(4): 253-268.

38. Trikha P, Carson WE (2014). Signaling pathways involved in MDSC regulation. Biochim Biophys Acta 1846: 5565.

39. Vasquez-Dunddel D, Pan F, Zeng $Q$, et al (2013). STAT3 regulates arginase-I in myeloid derived suppressor cells from cancer patients. J Clin Inves. 123: 1580-1589.

40. Lu T, Gabrilovich DI (2012). Molecular pathways: tumor-infiltrating myeloid cells and reactive oxygen species in regulation of tumor microenvironment. Clin Cancer Res. 18: 4877-4882.

41. Munder M, Engelhardt M, Knies D, et al (2013). Cytotoxicity of tumor antigen specific human T cells is unimpaired by arginine depletion. PLoS One. 8: e63521

42. Salman M. Toor, Azharuddin Sajid Syed Khaja, Haytham El Salhat, Issam Faour, Jihad Kanbar, Asif A. Quadri, Mohamed Albashir, Eyad Elkord (2017). Myeloid cells in circulation and tumor microenvironment of breast cancer patients. Cancer Immunol Immunother. 66(6): 753-764.

43. Pinton L, Solito S, Damuzzo V, et al (2016). Activated T cells sustain myeloid-derived suppressor cell-mediated immune suppression. Oncotarget. 7: 1168-1184.

44. Teo ZL, Versaci S, Dushyanthen S, et al (2017). Combined CDK4/6 and PI3Kalpha Inhibition Is Synergistic and Immunogenic in Triple-Negative Breast Cancer. Cancer Res. 77: 6340-6352.

45. Clavijo PE, Moore EC, Chen J, et al (2017). Resistance to CTLA-4 checkpoint inhibition reversed through selective elimination of granulocytic myeloid cells. Oncotarget. 8: 55804-55820. 
TABLES

\begin{tabular}{|c|c|c|c|c|c|c|}
\hline \multicolumn{7}{|c|}{ MDSC } \\
\hline & \multicolumn{2}{|c|}{ Total } & \multicolumn{2}{|c|}{ M-MDSC } & \multicolumn{2}{|c|}{ G-MDSC } \\
\hline & Median & IR & Median & IR & Median & IR \\
\hline$A B C$ & 35.43 & 180.73 & 18.46 & 30.67 & 11.38 & 57.60 \\
\hline HW & 17.53 & 16.96 & 10.28 & 17.48 & 4.99 & 16.96 \\
\hline$p$ value & \multicolumn{2}{|c|}{0.001} & \multicolumn{2}{|c|}{0.035} & \multicolumn{2}{|c|}{0.004} \\
\hline \multicolumn{7}{|c|}{$0 x-40+$} \\
\hline & \multicolumn{2}{|c|}{ Total } & \multicolumn{2}{|c|}{ CD4+OX-40 } & \multicolumn{2}{|c|}{ CD8+OX-40 } \\
\hline & Median & IR & Median & IR & Median & IR \\
\hline$A B C$ & 9.89 & 28.71 & 7.36 & 26.33 & 1.64 & 2.14 \\
\hline HW & 3.01 & 3.10 & 2.81 & 2.83 & 0.28 & 0.26 \\
\hline$p$ value & \multicolumn{2}{|c|}{$<0.001$} & \multicolumn{2}{|c|}{$<0.001$} & \multicolumn{2}{|c|}{$<0.001$} \\
\hline \multicolumn{7}{|c|}{ PD-1+ } \\
\hline & \multicolumn{2}{|c|}{ Total } & \multicolumn{2}{|c|}{ CD4+ PD-1+ } & \multicolumn{2}{|c|}{ CD8+ PD-1+ } \\
\hline & Median & IR & Median & IR & Median & IR \\
\hline$A B C$ & 10.77 & 8.55 & 6.95 & 5.14 & 3.63 & 2.57 \\
\hline HW & 13.00 & 6.19 & 10.04 & 5.27 & 3.18 & 2.47 \\
\hline$p$ value & \multicolumn{2}{|c|}{0.245} & \multicolumn{2}{|c|}{0.156} & \multicolumn{2}{|c|}{0.591} \\
\hline \multicolumn{7}{|c|}{ Treg } \\
\hline & \multicolumn{3}{|c|}{ Median } & \multicolumn{3}{|c|}{ IR } \\
\hline$A B C$ & \multicolumn{3}{|c|}{32.05} & \multicolumn{3}{|c|}{29.84} \\
\hline HW & \multicolumn{3}{|c|}{22.61} & \multicolumn{3}{|c|}{13.57} \\
\hline$p$ value & \multicolumn{6}{|c|}{0.001} \\
\hline
\end{tabular}

Table 1. Basal medians of MDSC, OX-40+, PD-1+ and regulatory T lymphocytes levels (cells/ul) in ABC patients and HW. MDSC, myeloid derived suppressor cells; M-MDSC, monocytic MDSCs; G-MDSC, granulocytic MDSCs; Tregs, regulatory $T$ lymphocytes; $A B C$, advanced breast carcinoma; HW, healthy women; IR, interquartile range. 


\begin{tabular}{|c|c|c|c|c|c|c|}
\hline \multicolumn{7}{|c|}{ MDSC } \\
\hline & \multicolumn{2}{|c|}{ Total } & \multicolumn{2}{|c|}{ M-MDSC } & \multicolumn{2}{|c|}{ G-MDSC } \\
\hline & Median & IR & Median & IR & Median & IR \\
\hline Basal & 32.05 & 29.84 & 18.46 & 30.67 & 11.38 & 16.77 \\
\hline C3 & 19.79 & 16.75 & 7.09 & 12.54 & 4.03 & 9.64 \\
\hline C6 & 19.01 & 21.51 & 4.89 & 10.64 & 4.85 & 8.31 \\
\hline $\begin{array}{c}\text { Basal vs C6 } \\
\text { P value }\end{array}$ & \multicolumn{2}{|c|}{$<0.001$} & \multicolumn{2}{|c|}{$<0.001$} & \multicolumn{2}{|c|}{$<0.001$} \\
\hline \multicolumn{7}{|c|}{$0 \times-40+$} \\
\hline & \multicolumn{2}{|c|}{ Total } & \multicolumn{2}{|c|}{ CD4+OX-40 } & \multicolumn{2}{|c|}{ CD8+OX-40 } \\
\hline & Median & IR & Median & IR & Median & IR \\
\hline Basal & 9.89 & 28.71 & 7.36 & 26.33 & 1.64 & 2.14 \\
\hline C3 & 13.75 & 25.94 & 12.23 & 22.72 & 1.24 & 2.28 \\
\hline C6 & 35.10 & 45.56 & 30.17 & 41.34 & 2.46 & 4.97 \\
\hline $\begin{array}{c}\text { Basal vs C6 } \\
\text { P value }\end{array}$ & \multicolumn{2}{|c|}{0.014} & \multicolumn{2}{|c|}{0.007} & \multicolumn{2}{|c|}{0.034} \\
\hline \multicolumn{7}{|c|}{ PD-1+ } \\
\hline & \multicolumn{2}{|c|}{ Total } & \multicolumn{2}{|c|}{ CD4+ PD-1+ } & \multicolumn{2}{|c|}{ CD8+ PD-1+ } \\
\hline & Median & IR & Median & IR & Median & IR \\
\hline Basal & 10.77 & 8.55 & 6.95 & 5.14 & 3.62 & 2.56 \\
\hline C3 & 5.02 & 5.01 & 3.51 & 3.69 & 1.82 & 1.81 \\
\hline $\mathrm{C} 6$ & 5.17 & 3.90 & 2.93 & 3.33 & 1.61 & 1.12 \\
\hline $\begin{array}{c}\text { Basal vs C6 } \\
\text { P value }\end{array}$ & \multicolumn{2}{|c|}{$<0.001$} & \multicolumn{2}{|c|}{$<0.001$} & \multicolumn{2}{|c|}{$<0.001$} \\
\hline \multicolumn{7}{|c|}{ Treg } \\
\hline & \multicolumn{3}{|c|}{ Median } & \multicolumn{3}{|c|}{ IR } \\
\hline Basal & \multicolumn{3}{|c|}{32.05} & \multicolumn{3}{|c|}{29.84} \\
\hline C3 & \multicolumn{3}{|c|}{19.79} & \multicolumn{3}{|c|}{16.75} \\
\hline C6 & \multicolumn{3}{|c|}{19.01} & \multicolumn{3}{|c|}{21.51} \\
\hline $\begin{array}{c}\text { Basal vs C6 } \\
\text { P value }\end{array}$ & \multicolumn{6}{|c|}{0.022} \\
\hline
\end{tabular}

Table 2. Basal, C3 and C6 medians of MDSC, OX-40+, PD-1+ and regulatory T lymphocytes levels (cells/ul) in ABC patients. MDSC; myeloid derived suppressor cells; M-MDSC; monocytic MDSCs; G-MDSC: granulocytic MDSCS; Tregs, regulatory $T$ lymphocytes; $C 3$, cycle 3; C6, cycle 6; IR, interquartile range. 


\begin{tabular}{|c|c|c|c|c|c|c|c|c|c|c|c|c|}
\hline \multicolumn{13}{|c|}{ MDSC } \\
\hline & \multicolumn{4}{|c|}{ Total } & \multicolumn{4}{|c|}{ M-MDSC } & \multicolumn{4}{|c|}{ G-MDSC } \\
\hline & \multicolumn{2}{|c|}{ CB } & \multicolumn{2}{|c|}{ PD } & \multicolumn{2}{|c|}{ CB } & \multicolumn{2}{|c|}{ PD } & \multicolumn{2}{|c|}{ CB } & \multicolumn{2}{|c|}{ PD } \\
\hline & Median & IR & Median & IR & Median & IR & Median & IR & Median & $\mathbf{I R}$ & Median & IR \\
\hline Basal & 33.63 & 39.34 & 37.48 & 20.63 & 18.46 & 33.49 & 16.20 & 22.97 & 12.74 & 18.14 & 14.20 & 28.56 \\
\hline C3 & 16.54 & 12.88 & 25.32 & 31.26 & 7.09 & 12.90 & 7.50 & 12.24 & 3.23 & 6.70 & 5.63 & 20.09 \\
\hline C6 & 10.36 & 15.84 & 78.54 & 128.63 & 4.15 & 6.48 & 42.04 & 86.99 & 3.36 & 7.03 & 10.00 & 24.23 \\
\hline $\begin{array}{c}\text { Basal vs C6 } \\
\text { P value }\end{array}$ & \multicolumn{2}{|c|}{$<0.001$} & \multicolumn{2}{|c|}{0.176} & \multicolumn{2}{|c|}{$<0.001$} & \multicolumn{2}{|c|}{0.310} & \multicolumn{2}{|c|}{$<0.001$} & \multicolumn{2}{|c|}{0.612} \\
\hline $\begin{array}{c}\text { CB vs PD } \\
\text { (C6) } \\
\text { P value }\end{array}$ & \multicolumn{4}{|c|}{0.003} & \multicolumn{4}{|c|}{0.004} & \multicolumn{4}{|c|}{0.002} \\
\hline \multicolumn{13}{|c|}{ OX-40+ } \\
\hline & \multicolumn{4}{|c|}{ Total } & \multicolumn{4}{|c|}{ CD4+OX-40+ } & \multicolumn{4}{|c|}{ CD8+OX-40+ } \\
\hline & \multicolumn{2}{|c|}{ CB } & \multicolumn{2}{|c|}{ PD } & $\mathrm{Cl}$ & & PD & & CI & & PD & \\
\hline & Median & $\mathbf{I R}$ & Median & IR & Median & IR & Median & $\mathbf{I R}$ & Median & IR & Median & $\mathbf{I R}$ \\
\hline Basal & 9.89 & 28.64 & 11.71 & 36.9 & 8.32 & 25.46 & 6.14 & 35.77 & 1.85 & 2.37 & 1.40 & 1.75 \\
\hline C3 & 15.23 & 26.78 & 4.57 & 12.9 & 12.93 & 25.02 & 4.00 & 10.97 & 1.24 & 2.29 & 1.20 & 1.29 \\
\hline C6 & 37.28 & 43.54 & 5.91 & 57.62 & 33.22 & 40.49 & 2.22 & 45.44 & 2.63 & 5.72 & 1.75 & 3.33 \\
\hline $\begin{array}{c}\text { Basal vs C6 } \\
\text { P value }\end{array}$ & $0 . c$ & & & & 0.0 & & 0.86 & & 0.0 & & 0.91 & \\
\hline $\begin{array}{c}\text { CB vs PD } \\
\text { (C6) } \\
\text { P value } \\
\end{array}$ & & & 199 & & & 0.1 & 99 & & & & 19 & \\
\hline & & & & & PD-1+ & & & & & & & \\
\hline & & & otal & & & CD4+ & PD-1+ & & & CD8 & PD-1 & \\
\hline & СВ & & PD & & $\mathrm{Cl}$ & & PD & & CB & & PD & \\
\hline & Median & IR & Median & IR & Median & IR & Median & IR & Median & IR & Median & IR \\
\hline Basal & 11.16 & 8.99 & 11.03 & 11.18 & 6.95 & 5.08 & 5.95 & 8.58 & 3.62 & 2.45 & 4.51 & 4.34 \\
\hline C3 & 5.02 & 3.99 & 4.96 & 14.81 & 3.51 & 3.28 & 2.16 & 8.11 & 1.63 & 1.62 & 2.24 & 6.66 \\
\hline C6 & 4.82 & 3.74 & 7.64 & 4.55 & 2.93 & 3.12 & 5.98 & 4.86 & 1.51 & 1.05 & 1.80 & 3.72 \\
\hline $\begin{array}{c}\text { Basal vs C6 } \\
\text { P value }\end{array}$ & $<0$. & & & & $<0.0$ & & 0.49 & & $<0.0$ & & 0.23 & \\
\hline $\begin{array}{c}\text { CB vs PD } \\
\text { (C6) } \\
\text { P value }\end{array}$ & & & 058 & & & & 12 & & & & 40 & \\
\hline & & & & & Tregs & & & & & & & \\
\hline & & & & & & & & & PI & & & \\
\hline & & Mediar & & & IR & & & Median & & & IR & \\
\hline Basal & & 31.87 & & & 27.87 & & & 39.30 & & & 52.24 & \\
\hline C3 & & 20.79 & & & 15.01 & & & 17.63 & & & 38.37 & \\
\hline C6 & & 19.01 & & & 16.64 & & & 28.36 & & & 32.03 & \\
\hline $\begin{array}{c}\text { Basal vs C6 } \\
\text { P value }\end{array}$ & & & & & & & & & 0.8 & & & \\
\hline $\begin{array}{c}\text { CB vs PD } \\
\text { (C6) } \\
\text { P value }\end{array}$ & & & & & & 0.680 & & & & & & \\
\hline
\end{tabular}

Table 3. Basal, $C 3$ and $C 6$ medians of MDSC, OX-40+, PD-1+ and regulatory T lymphocytes levels (cells/ul) in ABC patients according to response. MDSC; myeloid derived suppressor cells; M-MDSC; monocytic MDSCs; G-MDSC: granulocytic MDSCs; Tregs, regulatory T lymphocytes; $C B$, clinical benefit; $P D$, progression of disease; $C 3$, cycle 3; C6, cycle 6; IR, interquartile range. 
FIGURES

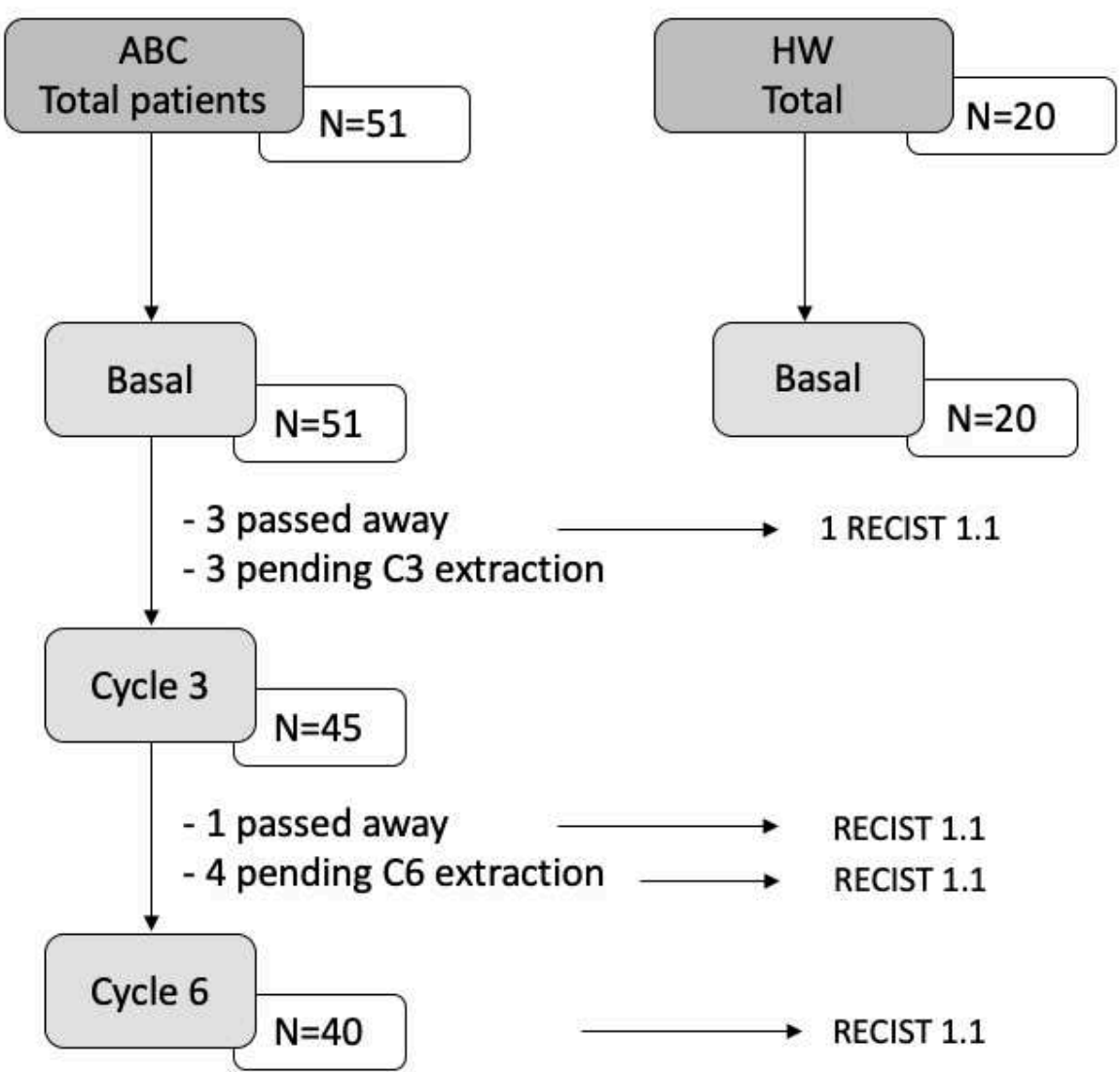

Figure 1. Diagram. HW, healthy women; ABC, advanced breast cancer; RECIST v1.1, response evaluation criteria in solid tumors. 

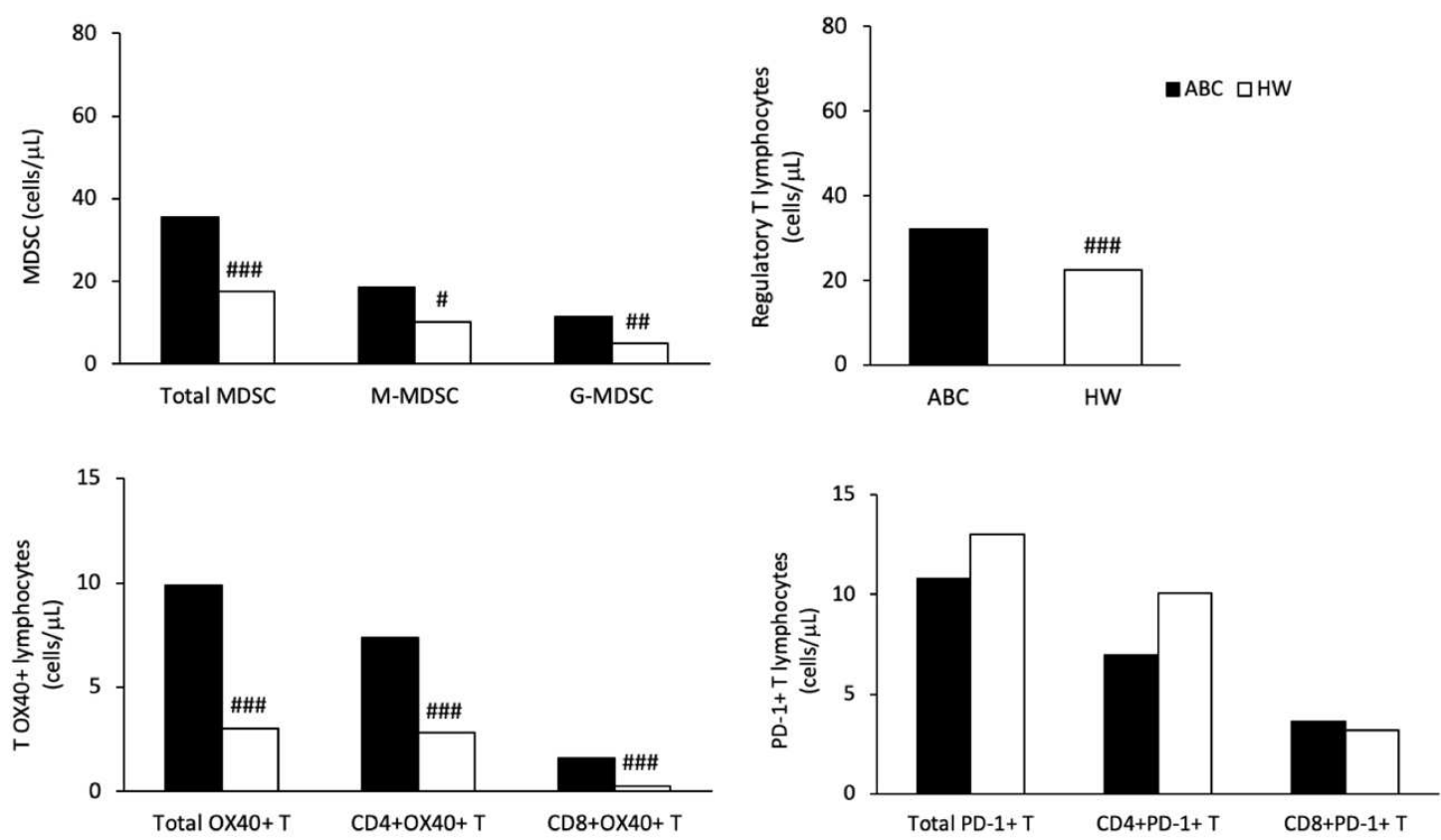

Figure 2. Basal medians of MDSC, OX-40+, PD-1+ and regulatory T lymphocytes levels (cells/ul) in ABC patients and HW. MDSC, myeloid derived suppressor cells; M-MDSC, monocytic MDSCs; G-MDSC, granulocytic MDSCs; $A B C$, advanced breast carcinoma; HW, healthy women. \#, \#\# and \#\#, $\mathrm{p} \leq 0.05, \mathrm{p} \leq 0.01$ and $\mathrm{p} \leq 0.001$ compared with $A B C$ patients. Different scales are used. 

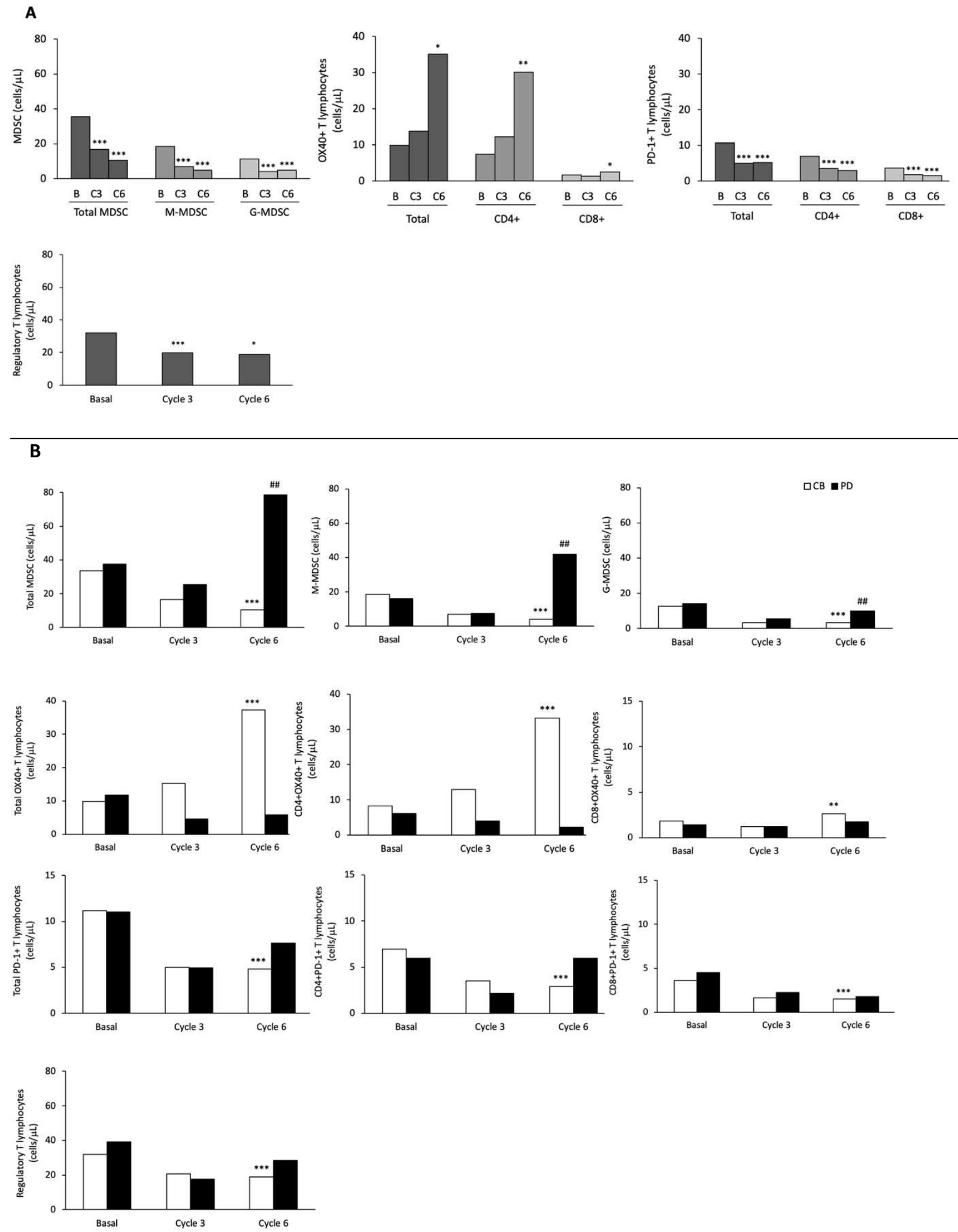

Figure 3. Basal, $C 3$ and $C 6$ medians of MDSCs, OX-40+, PD-1+ and regulatory T lymphocytes levels (cells/ul) in $A B C$ patients (A) according to response (B). MDSCs; myeloid derived suppressor cells; M-MDSC; monocytic MDSCs; G-MDSC: granulocytic MDSCs; Tregs, regulatory T lymphocytes; $C B$, clinical benefit; $P D$, progression of disease; C3, cycle 3; C6, cycle 6. ${ }^{*},{ }^{* *}$ and ${ }^{* * *}, \mathrm{p} \leq 0.05, \mathrm{p} \leq 0.01$ and $\mathrm{p} \leq 0.001$ compared with basal measurements, respectively. \#\#, p $\leq 0.01$ compared with CB patients. Different scales are used. 
Figures

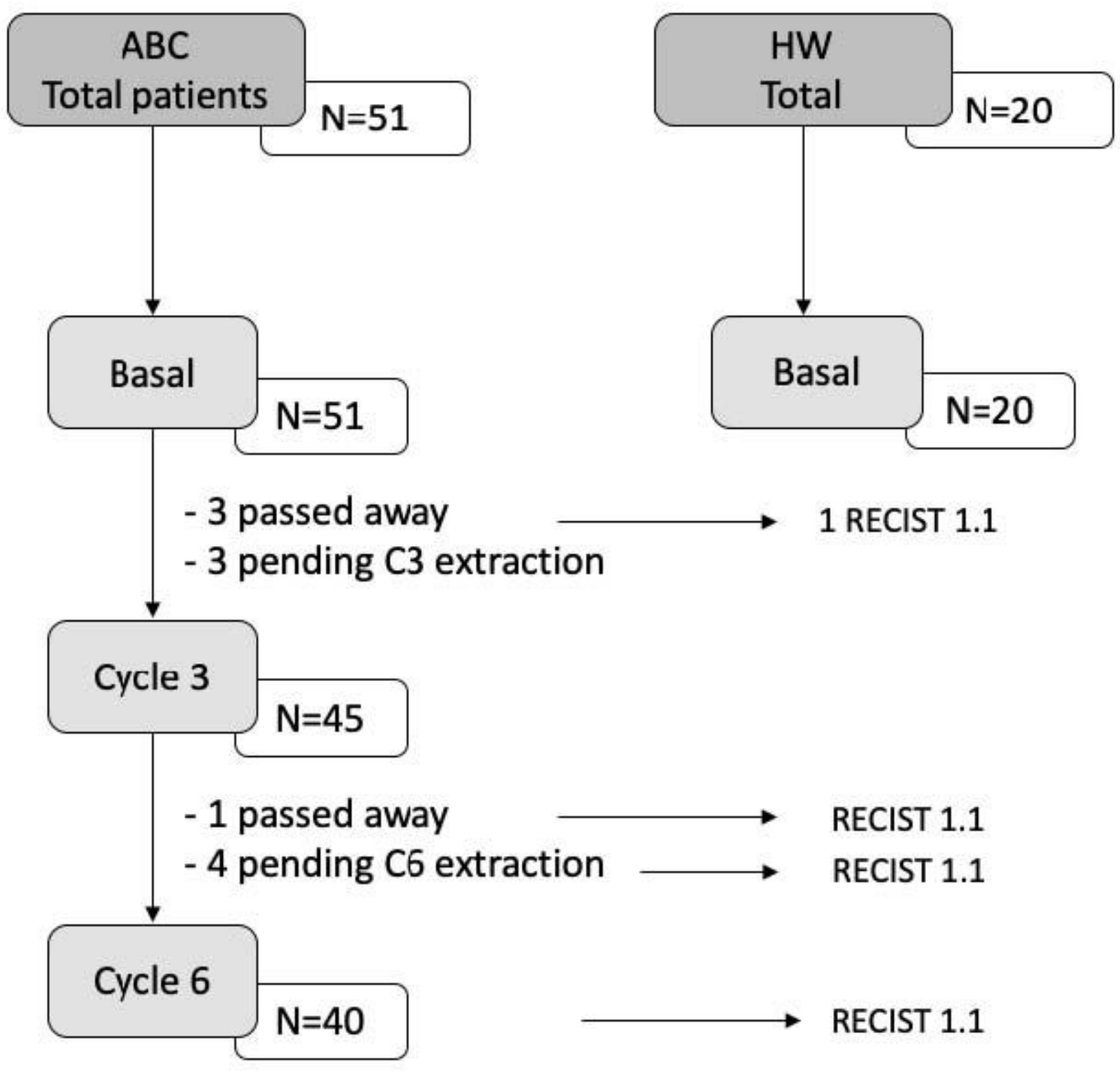

Figure 1

Diagram. HW, healthy women; ABC, advanced breast cancer; RECIST v1.1, response evaluation criteria in solid tumors. 

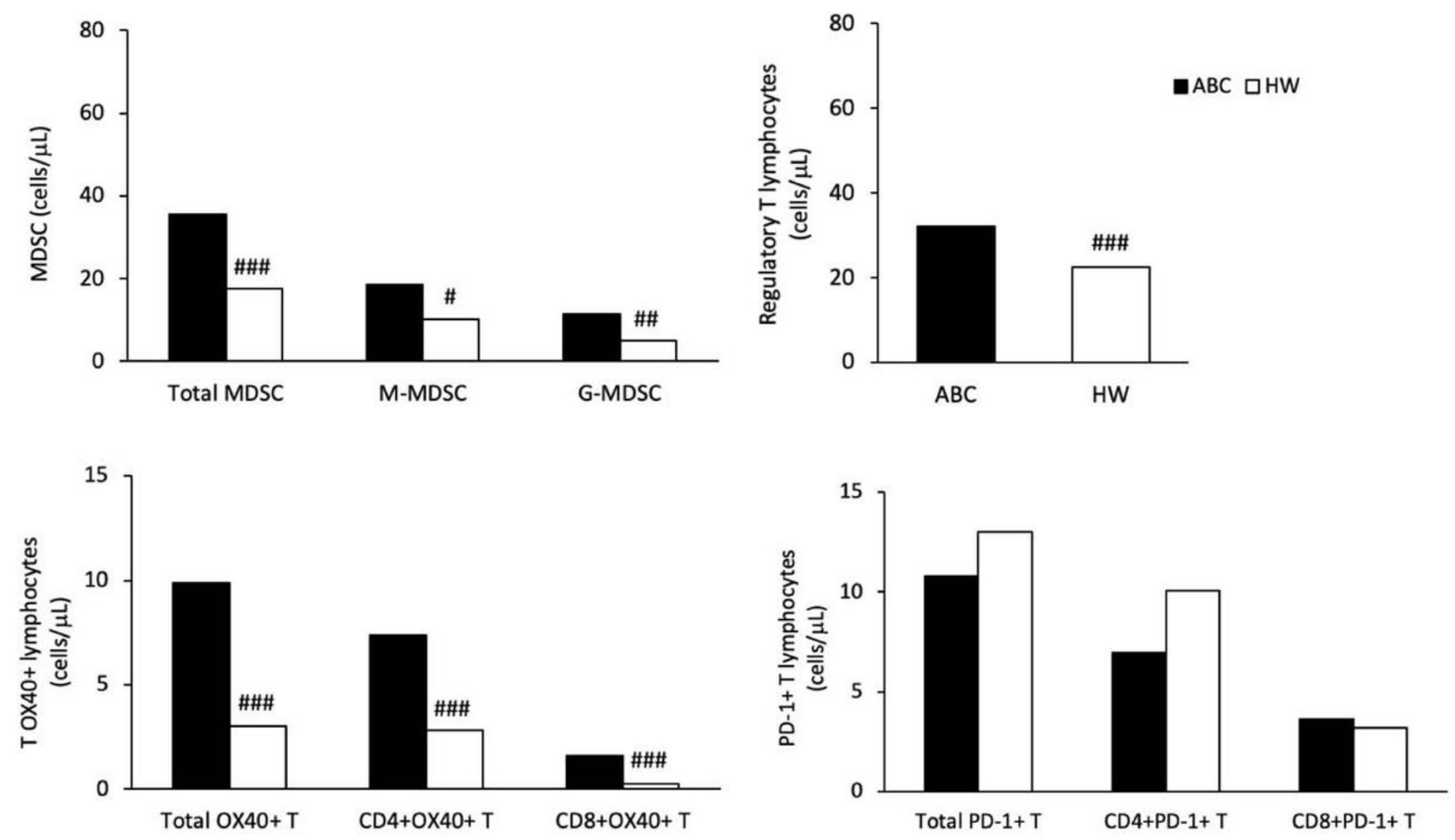

Figure 2

Basal medians of MDSC, OX-40+, PD-1+ and regulatory T lymphocytes levels (cells/ul) in ABC patients and HW. MDSC, myeloid derived suppressor cells; M-MDSC, monocytic MDSCs; G-MDSC, granulocytic MDSCs; ABC, advanced breast carcinoma; HW, healthy women. \#, \#\# and \#\#\#, $p \leq 0.05, p \leq 0.01$ and $p \leq 0.001$ compared with $A B C$ patients. Different scales are used. 

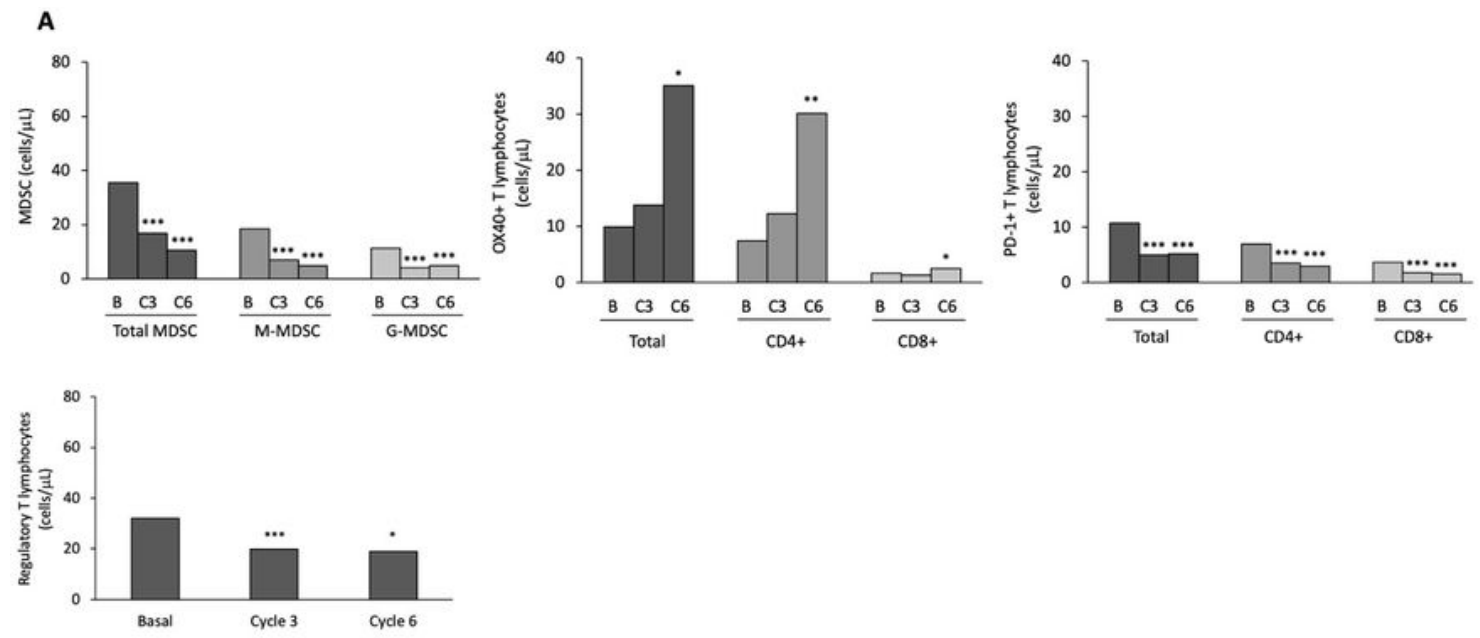

B
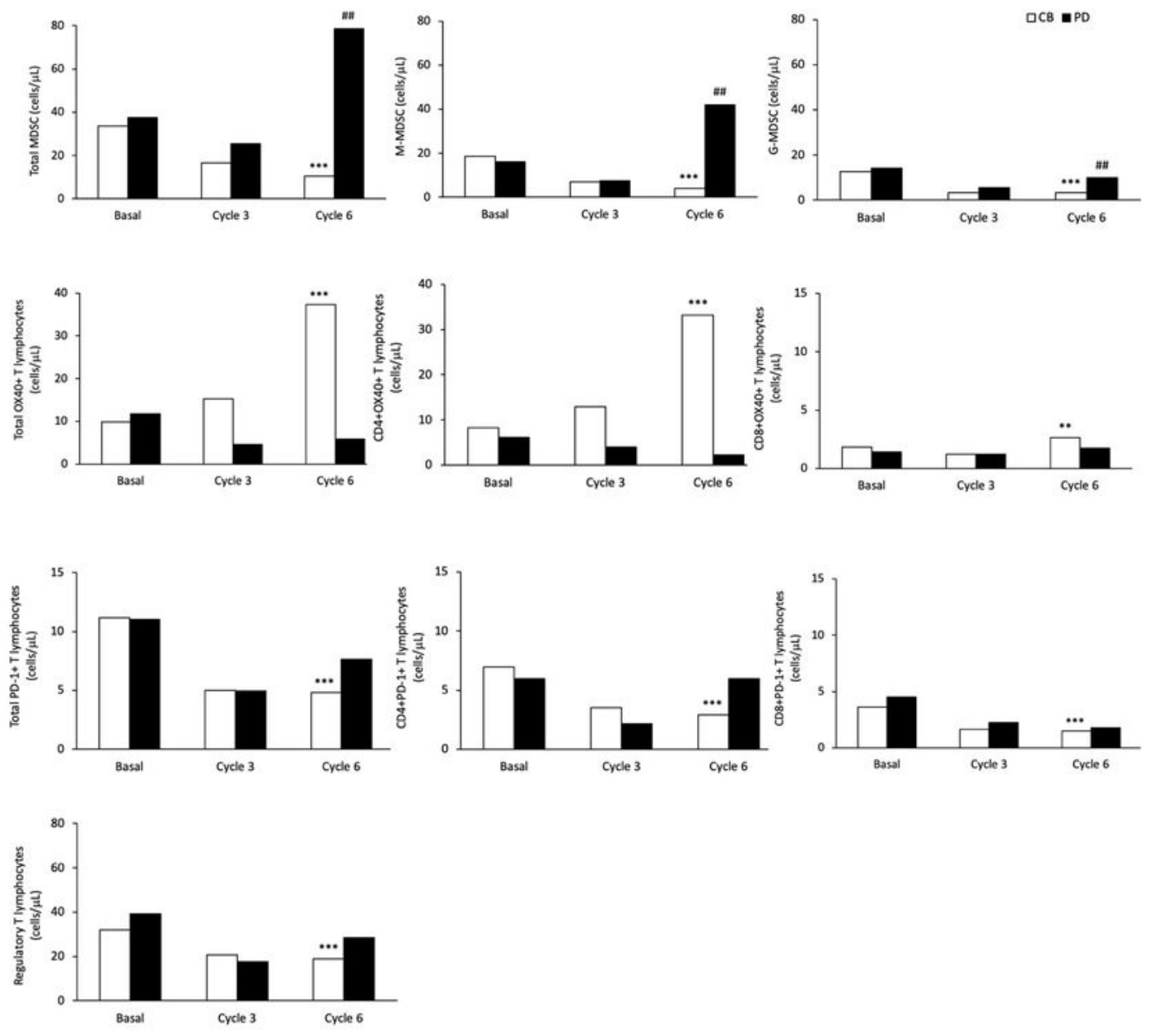

Figure 3

Basal, C3 and C6 medians of MDSCs, OX-40+, PD-1+ and regulatory T lymphocytes levels (cells/ul) in $A B C$ patients $(A)$ according to response (B). MDSCs; myeloid derived suppressor cells; M-MDSC; monocytic MDSCs; G-MDSC: granulocytic MDSCs; Tregs, regulatory T lymphocytes; CB, clinical benefit; $\mathrm{PD}$, progression of disease; $\mathrm{C} 3$, cycle $3 ; \mathrm{C} 6$, cycle 6 . *, ${ }^{\star *}$ and ${ }^{\star \star \star}, \mathrm{p} \leq 0.05, \mathrm{p} \leq 0.01$ and $\mathrm{p} \leq 0.001$ compared with basal measurements, respectively. \#\#, $\mathrm{p} \leq 0.01$ compared with CB patients. Different scales are used. 